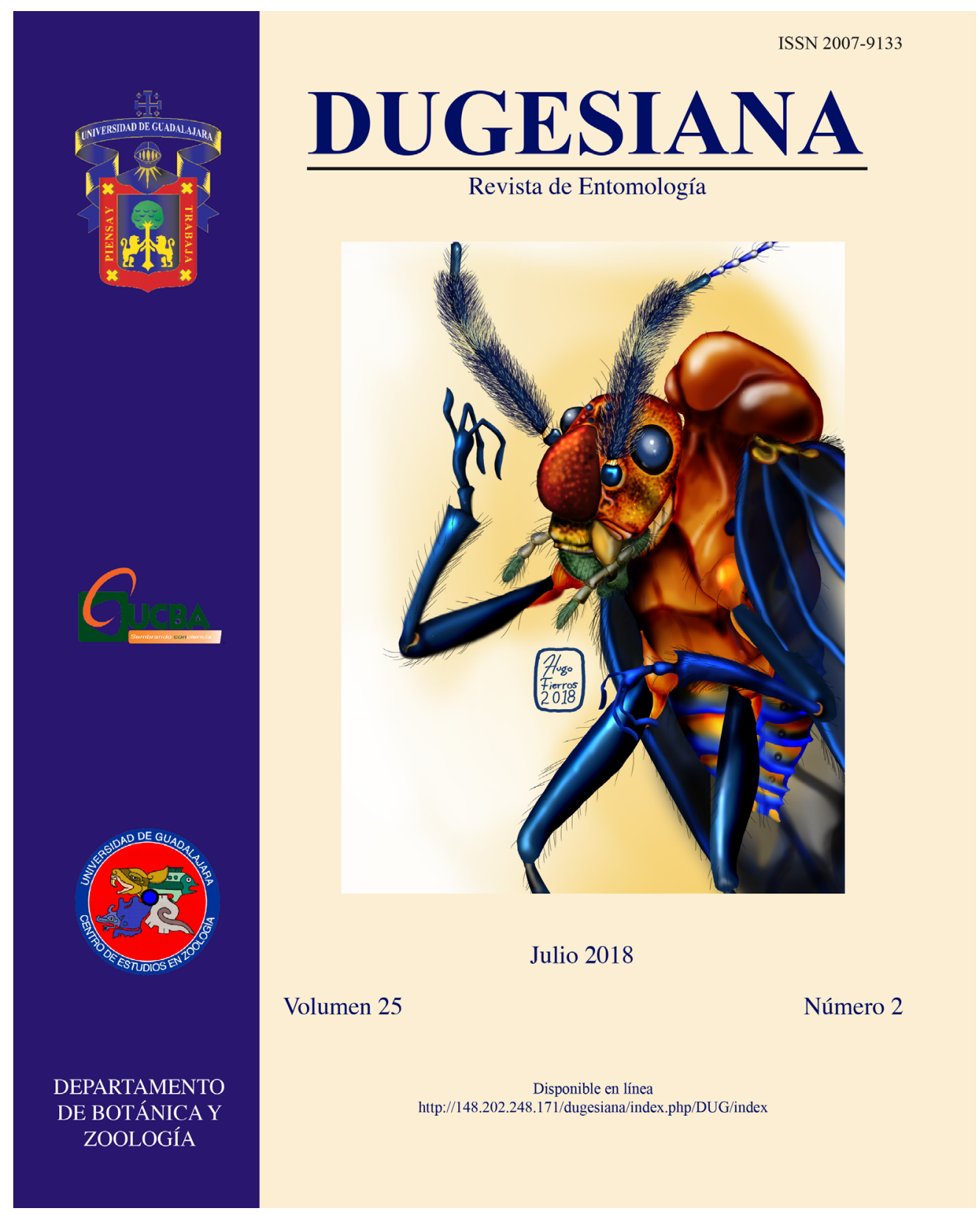

Dugesiana, Año 25, No. 2, julio 2018-diciembre 2018 (segundo semestre de 2018), es una publicación semestral, editada por la Universidad de Guadalajara, a través del Centro de Estudios en Zoología, por el Centro Universitario de Ciencias Biológicas y Agropecuarias. Camino Ramón Padilla Sánchez \# 2100, Nextipac, Zapopan, Jalisco, Tel. 37771150 ext. 33218, http://148.202.248.171/dugesiana/index.php/DUG/index, glenusmx@gmail.com. Editor responsable: José Luis Navarrete Heredia. Reserva de Derechos al Uso Exclusivo 04-2009-062310115100203, ISSN: 2007-9133, otorgados por el Instituto Nacional del Derecho de Autor. Responsable de la última actualización de este número: José Luis Navarrete Heredia, Editor y Ana Laura González-Hernández, Asistente Editorial. Fecha de la última modificación 25 de julio 2018, con un tiraje de un ejemplar.

Las opiniones expresadas por los autores no necesariamente reflejan la postura del editor de la publicación.

Queda estrictamente prohibida la reproducción total o parcial de los contenidos e imágenes de la publicación sin previa autorización de la Universidad de Guadalajara. 


\title{
Distribución de la familia Passalidae (Coleoptera: Scarabaeoidea) en un gradiente altitudinal en el Soconusco, Chiapas, México
}

\section{Passalidae (Coleoptera: Scarabaeoidea) distribution along an altitudinal gradient in the Soconusco, Chiapas, Mexico}

\author{
Eduardo R. Chamé-Vázquez ${ }^{1 *}$, Pedro Reyes-Castillo ${ }^{2}$, Benigno Gómez y Gómez ${ }^{3}$ y Guillermo \\ Ibarra-Núñez ${ }^{1}$
}

${ }^{1}$ El Colegio de la Frontera Sur (ECOSUR), Unidad Tapachula. Carretera Antiguo Aeropuerto km. 2.5, 30700 Tapachula, Chiapas, México. ${ }^{2}$ Instituto de Ecología, A.C., km. 2.5 Antigua Carretera a Coatepec No. 351, Congregación El Haya, 91070 Xalapa, Veracruz, México. ${ }^{3}$ El Colegio de la Frontera Sur (ECOSUR), Unidad San Cristóbal. Carretera Panamericana y Periférico Sur s/n. Barrio de María Auxiliadora, 29290, San Cristóbal de las Casas, Chiapas, México. *Autor de correspondencia: echame@ecosur.mx

\begin{abstract}
RESUMEN
El objetivo del presente trabajo fue conocer la distribución de las especies de Passalidae en un gradiente altitudinal en el Soconusco y relacionar la disponibilidad y características de la madera muerta con la riqueza y abundancia de pasálidos. Se seleccionaron nueve sitios con diferente altura sobre el nivel del mar (10-3000 m) y se usaron cinco transectos lineales de $50 \mathrm{~m}$ para la toma de datos en cada uno de los sitios. Cada transecto tuvo cinco puntos de muestreo, donde se midió diámetro y largo de la madera, estado de descomposición y presencia de pasálidos. Para determinar diferencias estadísticas se aplicó una prueba de Kruskal-Wallis para el volumen de madera y tablas de contingencia para la asociación de las tribus de pasálidos con los diferentes estados de descomposición. En total se registraron 15 especies de Passalidae correspondientes a nueve géneros y dos tribus de la subfamilia Passalinae. Se examinaron 224 piezas de madera con un volumen total de $10.54 \mathrm{~m}^{3}$, observándose una colonización del $13.8 \%$ de las piezas encontradas. La distribución de pasálidos fue diferente en todo el gradiente altitudinal, mostrando una alta riqueza y abundancia en dos sitios con hábitat conservado (bosque mesófilo de montaña). El volumen de madera muerta fue estadísticamente diferente entre los sitios, destacando los dos sitios $(1500$ y $2220 \mathrm{~m})$ por tener el mayor volumen de madera (principalmente del estado III). Se observó que el estado de descomposición sí determinó la colonización de los Passalidae, agrupando la mayoría de las especies los estados II y III. El microhabitat mayormente registrado fue el alboduramícola. Se observó que las prácticas agronómicas influyen sobre la comunidad de pasálidos, principalmente en los sitios que tuvieron cultivo de cacao y café.
\end{abstract}

Palabras clave: Volcán Tacaná, riqueza específica, transectos lineales, madera muerta.

\begin{abstract}
We studied the distribution of passalid beetles (Coleoptera: Passalidae) along an altitudinal gradient in Soconusco region, in Chiapas. We assessed the relation among the species diversity and abundance of passalid with the amount of dead wood and their dead host trees characteristics (successional age). We measured the volume and characteristics of all dead wood and the abundance of passalid in five sampling points per line transect (50m in length); five transects per locality in nine localities (10-3000 m). We inspected 224 rotten logs, branches or stumps (total volume $10.54 \mathrm{~m}^{3}$ ) and estimated a colonization rate of $13.8 \%$. We found 15 species (nine genera and two tribes) of Passalinae in the region and differences in diversity among sites. The highest richness and abundance of beetles were recorded in two sites with preserved forest (cloud forest). The amount $\left(\mathrm{m}^{3}\right)$ of dead wood was different among sites, with the two sites of cloud forest $(1500-2220 \mathrm{~m}$ ) with the highest volume (with a large proportion of dead wood of successional age III). The deadwood succession seems to drive the colonization of Passalidae, where successional age II and III hold more species of beetles. Some agronomic practices impact over the community of passalid beetles; the use of dead wood as fuel in rural communities, reduce the availability of deadwood in mass forest and therefore can disrupt some processes that occur only in the deadwood habitats.
\end{abstract}

Ke y words: Tacana volcano, species richness, line transects, dead wood.

Los pasálidos exhiben preferencias silvícolas, tendencias higrófilas, comportamiento subsocial y distribución cosmotropical (Reyes-Castillo 2000). Así mismo, destacan por su valor ecológico, siendo uno de los grupos de macrocoleópteros más importantes en la descomposición de madera muerta (Castillo y Morón 1992, Míss y Deloya 2007).
Desde la publicación de la obra "Monographie des Passales" de Percheron en 1835, donde se describe a los primeros pasálidos de México, el conocimiento actual sobre estos escarabajos se ha incrementado de manera significativa, lo cual ha permitido demostrar que México posee una alta riqueza genérica y específica, además de tener un elevado número de especies endémicas (ReyesCastillo 2002). Aunque se citan 93 especies en el territorio 
nacional (Beza-Beza et al. 2017, Delgado y MoraAguilar 2014, Jiménez-Ferbans y Reyes-Castillo 2014, Reyes-Castillo y Chamé-Vázquez 2014, Reyes-Castillo y Gutiérrez-Velázquez 2016), estimamos que la cifra podría aumentar debido al número de géneros y especies que faltan por describir.

Para Chiapas se conocen 49 especies y 19 géneros, colocándolo como el estado con mayor diversidad de Passalidae de México (Reyes-Castillo y GutiérrezVelázquez 2016). También existe un elevado número de especies endémicas $(17 \%)$ y se debe a que el estado forma parte del Núcleo Centroamericano, lugar donde se encuentra uno de los mayores sitios de endemismo del mundo (Schuster et al. 2003). Sin embargo, en Chiapas únicamente existen cinco estudios faunísticos que incluyen a la familia Passalidae (Chamé-Vázquez et al. 2007, Morón et al. 1985, Morón et al. 1988, Palacios et al. 1990, Valenzuela-González 1986), por lo que la fauna del estado ha sido estudiada fragmentadamente mediante trabajos monográficos, revisiones genéricas y descripciones de nuevas especies (Boucher 1988 y 2005, Castillo y ReyesCastillo 1984, Gillogly 2005, Jiménez-Ferbans y ReyesCastillo 2014, Quintero y Reyes-Castillo 1983, ReyesCastillo 1970, 1978, Reyes-Castillo y Castillo 1986, ReyesCastillo y Chamé-Vázquez 2014, Reyes-Castillo y Schuster 1983, Reyes-Castillo et al. 1987, Schuster 1991, Schuster y Reyes-Castillo 1990, Schuster et al. 2003 y 2005).

Indudablemente, aún queda mucho por hacer en el estudio de la familia Passalidae, no solo desde el punto de vista taxonómico y faunístico, sino también conocer los aspectos ecológicos que determinan la distribución de estas especies en cada una de las comunidades y como se relacionan entre ellas. Por ejemplo, se ha observado que la diversidad de este grupo de escarabajos a lo largo de un gradiente altitudinal no responde a un solo factor (altura sobre el nivel del mar), sino que constituyen una serie de factores como son el patrón de distribución natural de las especies, tipo de vegetación, temperatura e impacto humano (Jankowski et al. 2008, MacVean y Schuster 1981, Schuster y Cano 2005).

Grove (2002) señala, que la disponibilidad de madera y la calidad en que esta se encuentra, son los elementos más importantes en la diversidad de los insectos saproxilófagos. El impacto humano puede tener su principal efecto sobre la disponibilidad y distribución de la madera muerta, aunque existen evidencias en las que ciertas especies o poblaciones son afectadas cuando los organismos desean colonizar nuevos sitios, ya que la distancia entre los hábitats disponibles puede ser mayor y el número de estos hábitats puede ser modificado (Galindo-Cardona et al. 2007). Por tal motivo, conocer la disponibilidad y las características del recurso que los pasálidos utilizan, podrá generar bases para el entendimiento del flujo de nutrientes dentro de un ecosistema (Míss y Deloya, 2007), con una posible aplicación en el manejo y uso sustentable de los bosques tropicales (Galindo-Cardona et al. 2007).
Este trabajo analiza la distribución de la familia Passalidae a lo largo de un gradiente altitudinal en el Soconusco, Chiapas, México, relacionando su riqueza con la disponibilidad y características de la madera muerta en los sitios de muestreo.

\section{MATERIAL Y MÉTODOS}

Área de estudio. La región del Soconusco está localizada en el sureste del estado de Chiapas y se compone de 16 municipios con una extensión total de $5475.5 \mathrm{~km}^{2}$. Tiene un rango altitudinal que va desde el nivel del mar en la Planicie Costera hasta los 4064 msnm en la Sierra Madre de Chiapas. Presenta una diversidad de climas a lo largo de su gradiente (cálido subhúmedo con lluvias en verano por debajo de los $800 \mathrm{~m}$; semicálido húmedo entre los 1000 y $1800 \mathrm{~m}$; templado húmedo con lluvias todo el año por arriba de los $2000 \mathrm{~m}$ ), con una temperatura media anual de 16-30 ${ }^{\circ} \mathrm{C}$. La precipitación fluctúa entre 1200 a $3000 \mathrm{~mm}$ durante la temporada de lluvias (mayo a octubre), y entre 5 a $800 \mathrm{~mm}$ durante la temporada de estiaje (noviembre a abril). Las principales comunidades vegetales presentes son selva alta perennifolia, selva mediana subcaducifolia, selva baja caducifolia, bosques de pino-encino, bosque mesófilo de montaña, vegetación ribereña y manglar, además de una amplia variedad de agroecosistemas como café, cacao, plátano, mango, caña de azúcar, maíz y palma de aceite. Las cuencas hidrográficas de la región son las del Río Huixtla y Río Suchiate (Arriaga et al. 2017, Fernández-Bello 2008).

Para el estudio se seleccionaron nueve sitios a lo largo del gradiente altitudinal en el Soconusco (10 a 3000 msnm), cada uno con una altitud distinta (Figura 1). Los sitios se ubicaron en diversas comunidades vegetales (manglar, cacao, cafetal bajo sombra, bosque mesófilo de montaña y bosque de pino-encino), aunque varios de ellos presentaron el mismo tipo de vegetación (ver Cuadro 1). Tres de los sitios (Benito Juárez El Plan, Chiquihuites y Papales) se encuentran dentro de la Reserva de la Biosfera Volcán Tacaná (REBITA). Puesto que los adultos están presentes durante todo el año, los muestreos se realizaron en el periodo de mayo a octubre de 2008.

Recolección de organismos. En cada sitio se realizaron cinco transectos lineales, los cuales fueron orientados de manera arbitraria, procurando una separación mínima de $250 \mathrm{~m}$ de cualquier otro transecto. Esta distancia mínima, además de las condiciones abruptas del terreno, permitieron asumir que cada uno de los transectos funcionaran como réplicas. Los transectos lineales tuvieron una longitud de $50 \mathrm{~m}$ en los que se establecieron cinco puntos de muestreos separados cada $10 \mathrm{~m}$ a partir del sitio de inicio. Una vez seleccionado cada punto de muestreo, se revisó la madera muerta que se encontró a un metro de distancia del punto de muestreo (ver detalles en Figura 2). Las razones que justificaron esta técnica fueron: 1) que un sitio se puede muestrear en un corto periodo de tiempo (2 - 4 días); 2) que el impacto sea menor sobre la fauna saproxilófaga, ya que al examinar la madera muerta en busca de pasálidos, se modifica la disponibilidad de microhábitats adecuados; y 3) 


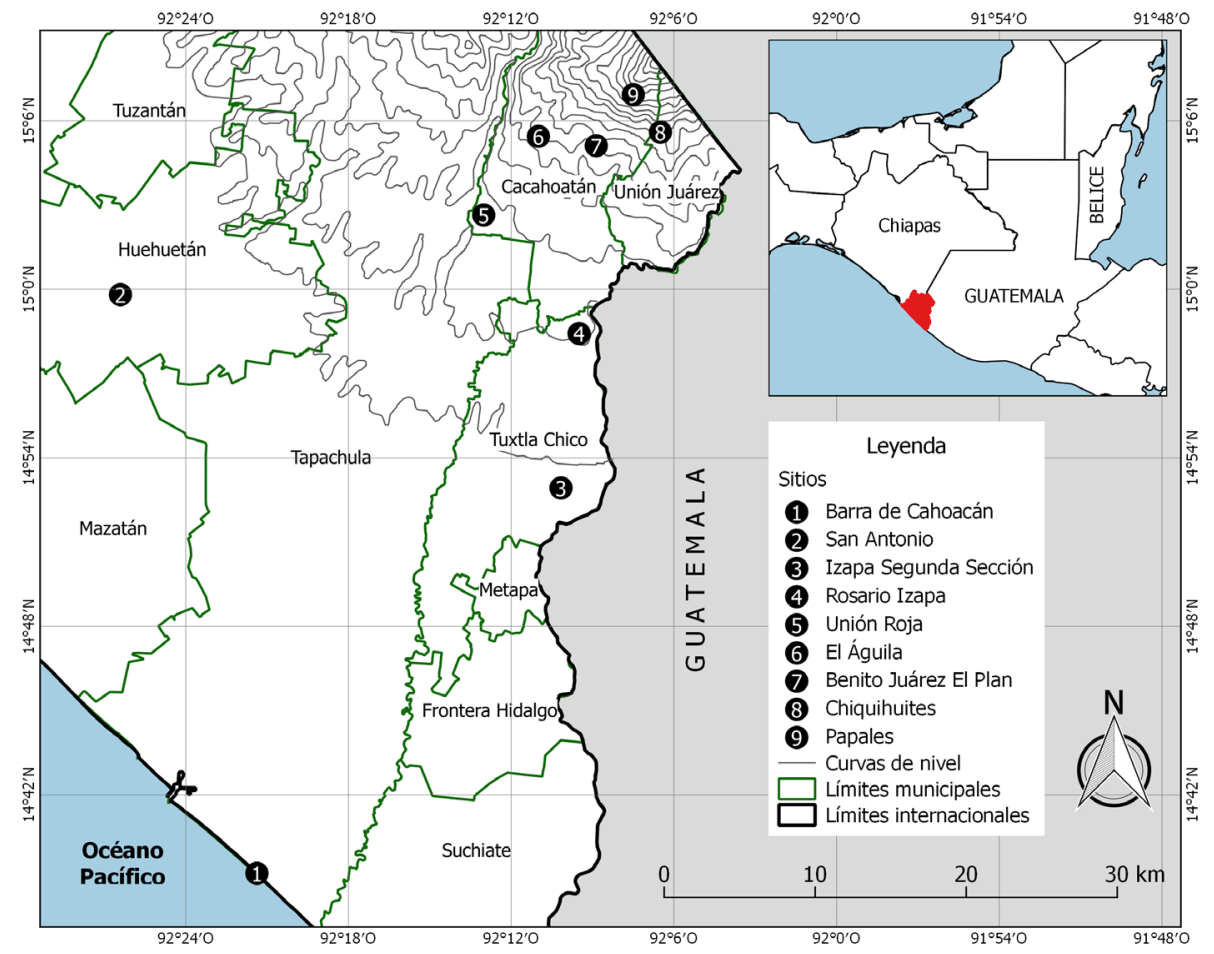

Figura 1. Ubicación de los sitios de muestreo ubicados a lo largo del gradiente altitudinal en el Soconusco, Chiapas. Los números asignados a cada sitio se indican en el Cuadro 1.

a)

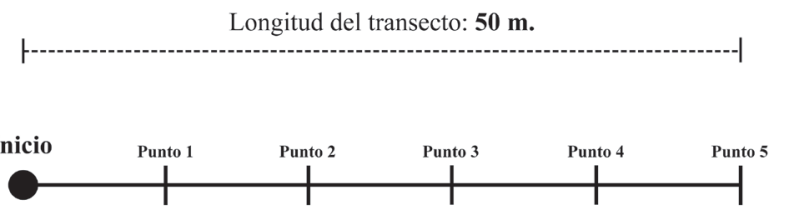

b)

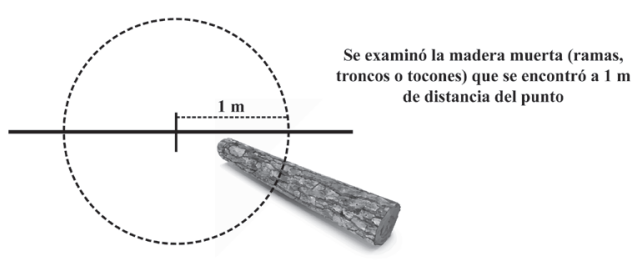

Figura 2. Uso de los transectos lineales: a) ubicación de los puntos de muestreos en el transecto, b) selección de la madera muerta con respecto al punto de muestreo.

que sea factible de realizarse en sitios donde los fragmentos de vegetación son de tamaño muy pequeños (e.g. parcelas de un agroecosistema).

En cada punto se revisó de manera minuciosa la madera muerta, clasificando el tipo de madera (tronco, rama o tocón), estado de descomposición (de acuerdo a los criterios de Castillo y Reyes-Castillo 2003), además de medir el largo y diámetro promedio (tomada en su longitud media) para el cálculo del volumen de madera muerta disponible. Se contabilizó el número de adultos por especie, mientras que la designación de los microhábitats se hizo conforme la explotación del recurso por parte de los pasálidos (subcortícolas a las especies debajo de la corteza, alboduramícolas si se hallaron en la parte interna de la madera, y si los organismos se encontraron entre la madera y el suelo se les clasificó como microhábitat interfase suelotronco, de acuerdo a lo señalado por Castillo y ReyesCastillo 1997). El material biológico fue depositado en la "Colección de Insectos asociados a plantas cultivadas en la Frontera Sur" (ECO-TAP-E) de El Colegio de la Frontera Sur, con duplicados en el Instituto de Ecología, A. C. (IEXA).

Análisis de datos. Los análisis se realizaron únicamente con la información de los adultos, ya que estos se pueden recolectar durante todo el año. Para probar diferencias estadísticas en el volumen de madera disponible entre los sitios se usó la prueba de Kruskal-Wallis con un nivel de significancia de 0.05. Mediante un análisis de tablas de contingencia se analizó la asociación de las dos tribus de la familia Passalidae (Passalini y Proculini) con respecto a los estados de descomposición, usando para ello un nivel de significancia de 0.05 . Los análisis se realizaron con el programa Statistica versión 7 (Statsoft Inc. 2004) y JMP versión 5.1 (SAS Inc. 2004).

\section{RESULTADOS}

En total se recolectaron 178 individuos adultos que representan a 15 especies, nueve géneros y dos tribus de la subfamilia Passalinae (Cuadro 2). Solamente se encontraron 13 nidos de ocho especies, que hicieron un total de 79 
larvas, 12 pupas y 25 huevos.

Los géneros Chondrocephalus Kuwert, 1896 y Passalus Fabricius, 1792 presentaron tres especies cada uno, siendo C. granulifrons (Bates, 1886) la especie con mayor abundancia en uno de los sitios y $P$. (P.) punctiger Lepeletier y Serville, 1825 la única especie que se encontró en cuatro sitios del gradiente altitudinal. Los sitios 7 (Benito Juárez), 8 (Chiquihuites) y 9 (Papales) son los que presentaron los valores más altos en cuanto a número de especies e individuos, siendo notorio que en el sitio 1 (Barra de Cahoacán) no se recolectaron pasálidos. Al analizar los tipos de hábitats del gradiente, el bosque mesófilo presentó la mayor cantidad de individuos (81\%) y especies de pasálidos (10).

A lo largo del gradiente se revisaron 224 piezas de madera muerta, las cuales sumaron $10.54 \mathrm{~m}^{3}$. La madera examinada tuvo un promedio en todo el gradiente de $12 \mathrm{~cm}$ de ancho, $1.65 \mathrm{~m}$ de largo y un volumen de $0.04 \mathrm{~m}^{3}$. La disponibilidad de madera muerta en cada uno de los sitios fue muy variable, con un promedio de 24.8 piezas por sitio. Los sitios 1 (Barra de Cahoacán) y 2 (San Antonio) presentaron el mayor número de piezas de madera, mientras que el sitio 5 (Unión Roja) tuvo la menor cantidad de ellas (Cuadro 3). Sin embargo, pese a la gran disponibilidad de madera a lo largo del gradiente, se obtuvo un promedio de $13.8 \%$ de colonización por parte de los pasálidos (Cuadro 3).

El volumen de la madera muerta fue estadísticamente diferente entre los sitios (prueba Kruskal-Wallis: $\mathrm{H}=$ 28.40, $\mathrm{P}=0.0004$ ). También se observó que los sitios mostraron diferencias en el volumen de madera por estado de descomposición. Los sitios 7 (Benito Juárez) y 8 (Chiquihuites) mostraron los mayores volúmenes de madera (2.17 y $2.69 \mathrm{~m}^{3}$, respectivamente), con una clara dominancia del estado III (Figura 3), lo cual a su vez coincidió con un mayor número de individuos y especies de pasálidos. Por otro lado, los sitios 2 (San Antonio) y 6 (El Águila) tuvieron una dominancia de madera en estado I, mientras que en el sitio 4 (Rosario Izapa) se observó una mayor presencia de madera en estados II y IV.

Es importante mencionar que el estado de descomposición de la madera sí determinó la colonización de los Passalidae, marcando diferencias estadísticas en las dos tribus estudiadas $\left(\mathrm{X}^{2}=10.11, \mathrm{gl}=3, \mathrm{P}=0.017\right)$. La tribu Passalini mostró una preferencia por el estado III y II, sin colonizar madera en estado IV. Por su parte, los Proculini fueron más dependientes del estado III y IV, aunque pueden colonizar madera en estado I y II. La especie Odontotaenius striatopunctatus se halló preferentemente en madera en estado I, mientras que Chondrocephalus granulifrons y Pseudacanthus subopacus prefirieron la madera en estado IV. Los estados II y III se encuentra colonizados por 12 especies de pasálidos, agrupando la mayor parte de las especies. El microhábitat mejor explotado fue el alboduramícola con el $97 \%$ de los individuos y la totalidad de especies. Por su parte, el uso del microhábitat subcortícola fue registrado en dos especies
(Passalus caelatus y Chondrocephalus gemmae), mientras que la interfase suelo-tronco en una sola especie (Passalus punctiger).

\section{DISCUSIÓN}

La fauna obtenida representa el $30 \%$ de las especies y el $47 \%$ de los géneros reportados para el estado de Chiapas. Para la región del Soconusco se ha reportado a Passalus (Passalus) interstitialis Eschscholtz, 1829, Ptichopus angulatus (Percheron, 1835), Chondrocephalus purulensis (Bates, 1886), Proculus goryi (Melly, 1833), Spurius bicornis (Truqui, 1857) y Verres hageni Kaup, 1871, los cuales no se encontraron en los transectos realizados (datos de la Colección IEXA y ECO-TAP-E).

Se determinó que el porcentaje de madera utilizada por los pasálidos es baja (13.8\%), ya que es una cifra menor al registrado en otras localidades de México (Los Tuxtlas, Veracruz y El Suspiro, Chiapas), Perú (Tingo María), Brasil (Manacapuru, Amazonas) y Puerto Rico (Bosque Experimental de Luquillo); no obstante, supera a otras localidades como Cañas en Costa Rica, Rio Cauca en Colombia y Xpujil en Campeche, México (GalindoCardona et al. 2007, Pardo-Locarno et al. 2000, Castillo y Reyes-Castillo 2003, Reyes-Castillo 2000). El porcentaje de colonización no fue el mismo en todo el gradiente, ya que el sitio 1 (Cahoacán) no registró a ninguna especie de pasálido. Este sitio tiene como vegetación dominante el manglar y no provee las condiciones necesarias para que los pasálidos puedan colonizar la madera muerta (ReyesCastillo et al. 2006), siendo reemplazados por las termitas (Blattodea) y hormigas (Hymenoptera: Formicidae) quienes ocuparon el $36 \%$ de los puntos de muestreo del sitio 1 y son consideradas como grupos de mayor importancia en la desintegración de la madera muerta (Morón y LópezMéndez 1985, Morón et al. 1988).

La variación en el ancho, largo y volumen de la madera de los sitios muestreados, podría estar determinada por el tipo de vegetación, grado de perturbación y prácticas forestales tradicionales, afectando no sólo a los pasálidos sino a toda la comunidad de insectos saproxilófagos (Míss y Deloya 2007). En el gradiente altitudinal destacaron los sitios 4 (Rosario Izapa), 7 (Benito Juárez) y 8 (Chiquihuites) con los valores más altos de volumen de madera sobre la media obtenida. Para el caso de Rosario Izapa, el cafetal bajo sombra se encontraba abandonado y no se observó una fuerte extracción de madera para leña, mientras que los otros dos sitios fueron hábitats conservados (bosque mesófilo), por lo que dichas comunidades vegetales ofrecen las condiciones adecuadas para la dispersión y colonización de los pasálidos. La preferencia de los pasálidos por madera de angiospermas y no tanto por gimnospermas, explicaría la baja presencia de este grupo en el Sitio 9 (Papales), en donde hay una clara dominancia de árboles del género Pinus (L.).

Lachat et al. (2006) indicaron que las tallas de la madera no son el único factor determinante, sino que también el 


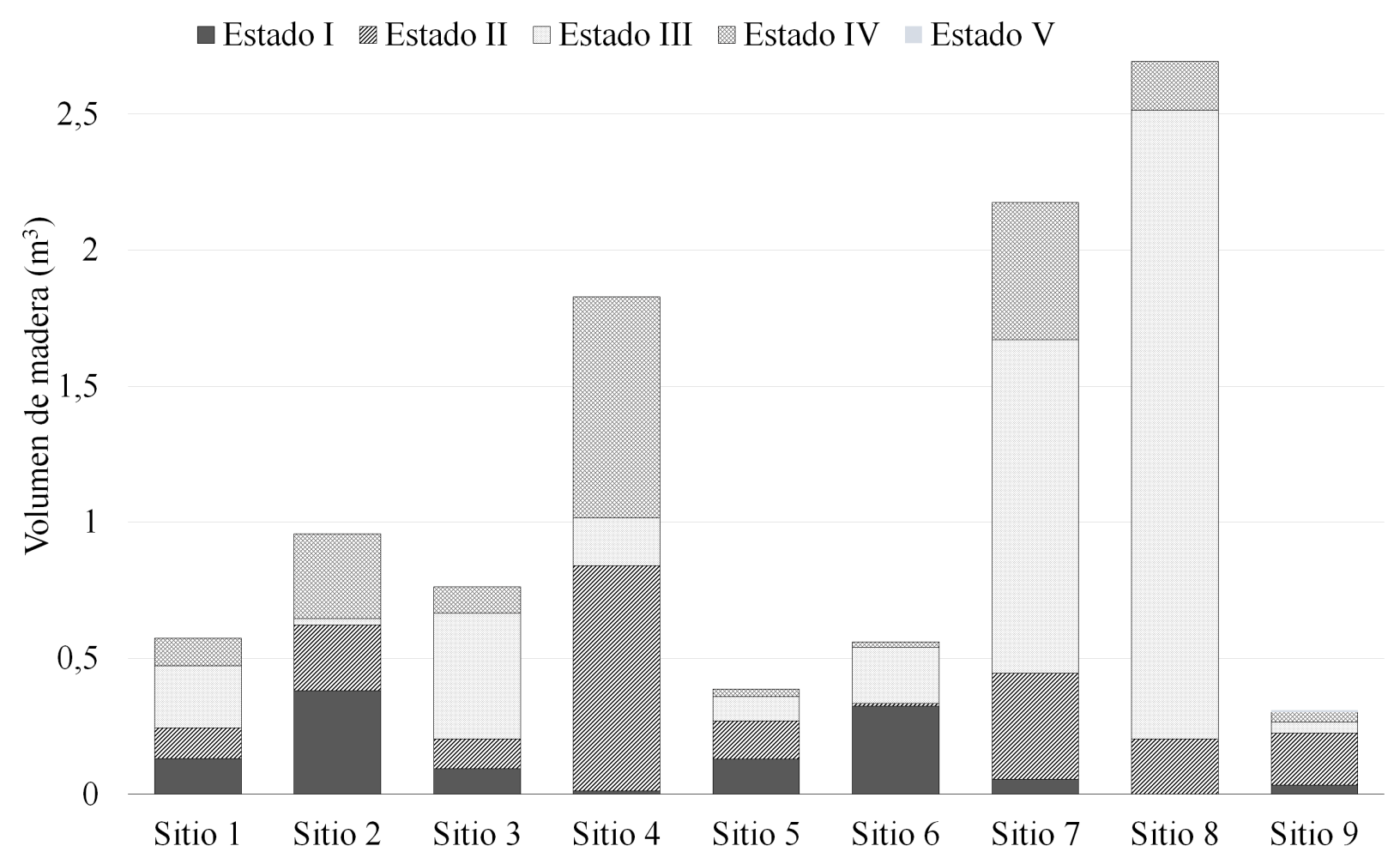

Figura 3. Volumen de madera examinada por estado de descomposición en los sitios de muestreo del gradiente altitudinal en el Soconusco.

estado de descomposición y su respectivo volumen, siendo aspectos muy importantes para el sostenimiento de la fauna saproxilófaga como los Passalidae. Se observó que en los sitios donde hubo una mayor cantidad de madera, mayor frecuencia de troncos, además de un mayor volumen en estados III y II, la diversidad de pasálidos se incrementó. Esto fue evidente en los sitios 7 (Benito Juárez El Plan) y 8 (Chiquihuites), que también compartieron el mismo tipo de vegetación (bosque mesófilo de montaña), uno de los hábitats más importantes para la fauna de pasálidos, tal como lo sugieren Castillo y Reyes-Castillo (2003).

Las observaciones de campo mostraron que una mayor presencia de ramas caídas en los sitios 2 (San Antonio), 3 (Izapa), 4 (Unión Roja) y 5 (Unión Roja) se debe al manejo agronómico que tienen los cacaotales y cafetales, ya que esa madera es el resultado de la poda de los cultivos, misma que es aprovechada por la gente local para su uso como leña. En estos sitios, la madera encontrada pertenecía a pocas especies de plantas, tales como café (Coffea arabica L.), mamey (Pouteria sapota (Jacq.) H.E. Moore y Stearn), cacao (Theobroma cacao L.), mango (Mangifera indica L.) y varias especies del genero Inga (Leguminosae), a diferencia de los sitios conservados donde la madera pertenecía a una mayor diversidad de árboles. La poca presencia de pasálidos en estos sitios también se relacionó a que prefieren colonizar principalmente troncos y no ramas o tallos muertos (Castillo y Reyes-Castillo 2003). De esta forma, se determina que los cambios en la estructura y composición de la vegetación original, pueden afectar a mediano y largo plazo la diversidad de los pasálidos en la región (Castillo y Lobo 2004). Es claro que el impacto humano ha jugado un papel muy importante en la diversidad de pasálidos, especialmente en sitios como el Soconusco, donde el cultivo extensivo de café y cacao han reemplazado grandes extensiones de bosques y selvas (Morón 1987).

En México, más del $90 \%$ de las poblaciones rurales utilizan la madera como combustible, que si bien no causa la deforestación de los bosques, si modifica los ecosistemas naturales (Masera 1993). Se ha observado que la madera muerta es recolectada sistemáticamente por las comunidades rurales, hasta el punto en que la materia vegetal muerta se agota y los pobladores derriban árboles vivos (Challenger 1998). Este fenómeno no se considera un caso aislado de nuestra zona de estudio, puesto que se han observado grandes volúmenes de madera que son extraídos por los pobladores a lo largo de todo el gradiente altitudinal. En los sitios conservados, la diversidad vegetal permite un buen reservorio de madera, ya que no todas las especies de plantas son preferidas para leña o uso en las comunidades, a diferencia de los sitios con cacaotales y cafetales, donde la baja diversidad de especies que ahí existen es porque los pobladores las seleccionan para sombra, alimento, leña o 
madera para construcción. Por tanto, los sitios con estos agroecosistemas tienen una fuerte extracción de madera y consecuentemente una fuerte presión sobre la fauna del suelo. Dado el efecto acumulativo de estas prácticas, la dinámica natural de la reintegración de los nutrientes al suelo está cambiando, causando inevitablemente un empobrecimiento de la biodiversidad (Lachat et al. 2006). Esto puede verse en la zona baja del Soconusco, donde los cultivos de mango y plátano son muy importantes y ocupan grandes extensiones, pero no ofrecen ningún recurso maderable a la fauna de Passalidae, motivo por el cual fueron descartados de nuestro estudio.

La importancia del área de estudio en cuanto a su biodiversidad (Micó et al. 2006; Schuster et al. 2000) sugiere que se realice un uso y manejo sustentable de los recursos naturales de la región. Afortunadamente, se cuenta con la presencia de la Reserva de la Biosfera Volcán Tacaná en la parte alta del gradiente, que si bien alberga una gran parte de la biodiversidad, aún excluye a elementos y procesos ecológicos de gran importancia. Es recomendable generar nuevos conocimientos, e integrar los ya existentes, para la conservación de la biodiversidad en el Soconusco.

\section{AGRADECIMIENTOS}

El primer autor agradece al Consejo Nacional de Ciencia y Tecnología (CONACYT) por la beca otorgada en los estudios de posgrado. Agradecemos a El Colegio de la Frontera Sur (ECOSUR, Unidad Tapachula) por las facilidades otorgadas para la realización de este trabajo y a las comunidades del Volcán Tacaná por su hospitalidad y compañía durante los muestreos. A David Chamé por la revisión y traducción del resumen al inglés, además de dos revisores anónimos que aportaron valiosas sugerencias a este manuscrito.

\section{LITERATURA CITADA}

Arriaga, L., V. Aguilar, J. Alcocer, R. Jiménez, E. Muñoz y E. Vázquez (coords). 2017. Regiones hidrológicas prioritarias. Comisión Nacional para el Conocimiento y Uso de la Biodiversidad. México. http://www.conabio. gob.mx/conocimiento/regionalizacion/doctos/rhp_032. html. Fecha de consulta: abril de 2018.

Beza-Beza, C.F., J. Beck, P. Reyes-Castillo y M. L. Jameson. 2017 Phylogeny of the genus Yumtaax Boucher (Coleoptera, Passalidae, Proculini): Taxonomic and evolutionary implications with descriptions of three new species. ZooKeys, 667: 95-129.

Boucher, S. 1988. Révision des espèces mexicaines du genre Veturius Kaup (Coleoptera: Passalidae). Annales de la Société Entomologique de France (n.s.) 24: 295305.

Boucher, S. 2005. Évolution et phylogénie des coléoptères Passalidae (Scarabaeoidea). Les taxons du groupe famille. La tribu neótropicale des Proculini et son complex Veturius. Annales de la Société Entomologique de France (n.s.) 41 (3-4): 239-604.
Castillo, C. y P. Reyes-Castillo. 1984. Biosistemática del género Petrejoides Kuwert (Coleoptera, Lamellicornia, Passalidae). Acta Zoológica Mexicana (n. s.) 4: 1-84.

Castillo, M. L. y J. M. Lobo. 2004. A comparison of Passalidae (Coleoptera, Lamellicornia) diversity and community structure between primary and secondary tropical forest in Los Tuxtlas, Veracruz, Mexico. Biodiversity and Conservation 13: 1257-1269.

Castillo M. L. y M. A. Morón. 1992. Observaciones sobre la degradación de madera por algunas especies de pasálidos (Coleoptera: Lamellicornia).

Folia Entomológica Mexicana, 84: 35-44.

Castillo, M. L. y P. Reyes-Castillo. 1997. Passalidae. (pp. 293-298). En González Soriano, E., R. Dirzo y R. Voght (Eds). Historia Natural de Los Tuxtlas, Veracruz, México. Instituto de Biología, UNAM. México.

Castillo, M. L. y P. Reyes-Castillo. 2003. Los Passalidae: coleópteros tropicales degradadores de troncos de árboles muertos. (pp. 237-262). En: Álvarez-Sánchez, J. y E. Naranjo-García (Eds.). Ecología del Suelo en la selva tropical húmeda de México. Instituto de Ecología, A. C., Instituto de Biología y Facultad de Ciencias, UNAM. Xalapa, Veracruz.

Challenger, A. 1998. Utilización y conservación de los ecosistemas terrestres de México: pasado, presente y futuro. Comisión Nacional para el Uso y Conocimiento de la Biodiversidad, Instituto de Biología de la UNAM y Agrupación Sierra Madre S. C., México.

Chamé-Vázquez, E. R., P. Reyes-Castillo y B. Gómez. 2007. Fauna de Passalidae (Coleoptera: Scarabaeoidea) en el bosque mesófilo de montaña del sureste de Chiapas, México. En: Zunino, M y A. Melic (Eds.). Escarabajos, diversidad y conservación biológica. m3m: Monografías Tercer Milenio 7: 63-68.

Delgado, L. y E. F. Mora-Aguilar. 2014. Proculus reyescastilloi Delgado and Mora-Aguilar (Coleoptera: Passalidae: Proculini): a new species from the region of Chimalapas, Oaxaca, Mexico. The Coleopterist Bulletin 68(1): 91-94.

Fernández-Bello, E., 2008. La producción agropecuaria en el Soconusco e intercambio con Centroamérica. (pp. 185200). En: J. E. Sánchez y R. Jarquin (Eds). La Frontera Sur. Reflexiones sobre el Soconusco, Chiapas, y sus problemas ambientales, poblacionales y productivos. México: Senado de la República-ECOSUR.

Galindo-Cardona, A., T. Giray, A. M. Sabat y P. ReyesCastillo. 2007. Bess beetle (Coleoptera: Passalidae); substrate availability, dispersal, and distribution in a subtropical wet forest. Annals of the Entomological Society of America 100: 711-720.

Gillogly, A. R. 2005. Review of the genus Popilius and preliminary phylogeny of Passalidae (Coleoptera). PhD Thesis. Texas A\&M University.

Grove, S. J. 2002. Saproxylic insect ecology and the sustainable management of forests. Annual Review of Ecology and Systematics 33: 1-23. 
Jankowski, J. E., A. L. Ciecka, N. Y. Meyer y K. N. Rabenold. 2008. Beta diversity along environmental gradients: implications of habitat specialization in tropical montane landscapes. Journal of Animal Ecology 78: 315-327.

Jiménez-Ferbans, L. y P. Reyes-Castillo. 2014. Phylogeny, biogeography and description of Ameripassalus, a new Mesoamerican genus of Passalidae (Coleoptera). Invertebrate Systematics 28(2): 124-144.

Lachat, T., P. Nagel, Y. Cakpo, S. Attignon, G. Goergen, B. Sinsin y R. Peveling. 2006. Dead wood and saproxylic beetle assemblages in a semi-deciduous forest in Southern Benin. Forest Ecology and Management, 225(1-3): 27-38.

MacVean, C. y J. C. Schuster. 1981. Altitudinal distribution of passalid beetles, Coleoptera Passalidae, and Pleistocene dispersal on the volcanic chain of northern Central America. Biotropica 13: 29-38.

Masera O. R., 1993. Sustainable fuel wood use in Rural Mexico. Volume I: current patterns of resource use. Lawrence Berkeley Laboratory, University of California.

Micó, E., B. Gómez y E. Galante. 2006. The Mesoamerican genus Yaaxkumukia: biogeography and description of new species (Coleoptera: Rutelidae). Annals of the American Entomological Society 99: 1-6.

Míss, D. J. V. y C. Deloya. 2007. Observaciones sobre los coleópteros saproxilófilos (Insecta: Coleoptera) en Sotuta, Yucatán, México. Revista Colombiana de Entomología, 33(1): 77-81.

Morón, M. A. 1987. The necrophagous Scarabaeinae beetles (Coleoptera: Scarabaeidae) from a coffee plantation in Chiapas, Mexico: habits and phenology. The Coleopterists Bulletin 41: 225-232.

Morón, M. A. y J. A. López-Méndez. 1985. Análisis de la entomofauna necrófila de un cafetal en el Soconusco, Chiapas, México. Folia Entomológica Mexicana 63: 47-59.

Morón, M. A., F. A. Villalobos y C. Deloya. 1985. Fauna de coleópteros lamelicornios de Boca del Chajul, Chiapas, México. Folia Entomológica Mexicana 66: 57-118.

Morón, M. A., J. Valenzuela y R. A. Terrón. 1988. La macrocoleopterofauna saproxilófila del Soconusco, Chiapas, México. Folia Entomológica Mexicana 74: 145-158.

Palacios, R. M., V. Rico y E. Fuentes. 1990. Inventario preliminar de los Coleoptera Lamellicornia de la zona de Yaxchilán, Chiapas, México. Folia Entomológica Mexicana 78: 49-60.

Pardo-Locarno, L. C., Lozano-Zambrano, F. H. y MontoyaLerma, J. 2000. Passalidae (Coleoptera: Scarabaeoidea) en fragmentos de bosque seco tropical de la cuenca media del Río Cauca, Colombia. Folia Entomológica Mexicana 110: 15-22.

Quintero, G. y P. Reyes-Castillo. 1983. Monografía del género Oileus Kaup (Coleoptera, Scarabaeoidea,
Passalidae). Folia Entomológica Mexicana 57:1-50.

Reyes-Castillo, P. 1970. Coleoptera, Passalidae: morfología y división en grandes grupos: géneros americanos. Folia Entomológica Mexicana 20-22: 1-240.

Reyes-Castillo, P. 1978. Revisión monográfica del género Spurius Kaup (Coleoptera, Passalidae). Studia Entomologica 20: 269-290.

Reyes-Castillo, P. 2000. Coleoptera Passalidae de México. (pp.171-182). En: Martín-Piera, F., J. J. Morrone y A. Melic (Eds). Hacia un proyecto CyTED para el inventario y estimación de la diversidad entomológica en Iberoamérica: PriBES-2000. m3m: Monografías Tercer Milenio, vol. 1, Sociedad Entomológica Aragonesa, Zaragoza, España.

Reyes-Castillo, P. 2002. Passalidae. (pp. 465-483). En: Llorente Bousquets, J. y J. J. Morrone (Eds.). Biodiversidad, Taxonomía y Biogeografía de Artrópodos: Hacia una síntesis de su conocimiento. Volumen III. Universidad Nacional Autónoma de México. México, D.F.

Reyes-Castillo, P. y C. Castillo. 1986. Nuevas especies de Coleoptera Passalidae de la Zona de Transición Mexicana. Anales del Instituto de Biología de la UNAM (serie Zoología) 56: 141-154.

Reyes-Castillo, P. y E. R. Chamé-Vázquez. 2014. Nueva especie mexicana de Pseudacanthus Kaup (Coleoptera: Passalidae). Dugesiana 21(2): 175-178.

Reyes-Castillo, P. y A. Gutiérrez-Velázquez. 2016. Familias Lucanidae Latreille, 1804 y Passalidae Leach, 1815. (pp. 55-67 + láminas 8-12 y 48). En: Deloya, C., J. Ponce Saavedra, P. Reyes-Castillo y G. Aguirre-León (Eds.). Escarabajos del Estado de Michoacán (Coleoptera: Scarabaeoidea). Primera edición, Universidad Michoacana de San Nicolás de Hidalgo, México.

Reyes-Castillo, P. y J.C. Schuster. 1983 Notes on some Mesoamerican Passalidae (Coleoptera): Petrejoides and Pseudacanthus. The Coleopterists Bulletin 37: 49-54.

Reyes-Castillo, P., C. R. V. Da Fonseca y C. Castillo. 1987. Descripción de un nuevo género mesoamericano de Passalidae (Coleoptera: Lamellicornia). Folia Entomológica Mexicana 73: 47-67.

Reyes-Castillo, P., C. V. Rojas-Gómez y H. Vázquez. 2006. Patrones de distribución de la familia Passalidae (Coleoptera). (pp. 237-270). En: Morrone, J. J. y J. Llorente-Bousquets (Eds). Componentes bióticos principales de la Entomofauna Mexicana. Las Prensas de Ciencias, UNAM. México, D.F.

SAS Institute. 2004. JMP Statistical Discovery Software. Versión 5.1. USA.

Schuster, J. C. 1991. Petrejoides (Col.: Passalidae): four new species from Mesoamerica and Mexico with a key to the genus. Florida Entomologist 74: 422-432.

Schuster, J. C. y E. B. Cano. 2005. La distribución mesoamericana de montaña: Síntesis de Passalidae (Coleoptera: Scarabaeoidea) para Mesoamérica Nuclear. (pp. 257-268). En: Llorente, J. y J. J. Morrone 
(Eds). Regionalización biogeográfica en Iberoamérica $\mathrm{y}$ tópicos afines. Facultad de Ciencias, Universidad Nacional Autónoma de México.

Schuster, J. C. y P. Reyes-Castillo. 1990. Coleoptera, Passalidae: Ogyges Kaup, Revisión de un género mesoamericano de montaña. Acta Zoológica Mexicana (n. s.) 40: 1-49.

Schuster, J. C., E. B. Cano y C. Cardona. 2000. Un método sencillo para priorizar la conservación de los bosques nubosos de Guatemala, usando Passalidae (Coleoptera) como organismos indicadores. Acta Zoológica Mexicana (n. s.) 80: 197-209.

Schuster, J. C., E. B. Cano y P. Reyes-Castillo. 2003. Proculus, giant Latin-American passalids: a generic revision, phylogeny and biogeography. Acta Zoológica
Mexicana (n. s.) 90: 281-306.

Schuster, J. C., E. B. Cano y S. Boucher. 2005. Ogyges and Veturius (Coleoptera: Passalidae) in Central America: synonymies, range extensions and new species. Acta Zoológica Mexicana (n. s.) 21: 115-132.

StatSoft, Inc. 2004. STATISTICA (data analysis software system), version 7. http:www.statsoft.com. Citada 26 Septiembre 2008

Valenzuela-González, J. 1986. Life cycle of the subsocial beetle Heliscus tropicus (Coleoptera: Passalidae) in a tropical locality in Southern México. Folia Entomológica Mexicana 68: 41-51.

Recibido: 12 de agosto 2017

Aceptado: 27 de abril 2018

Cuadro 1. Sitios de muestreos de Passalidae localizados en un gradiente altitudinal en la región del Soconusco, Chiapas.

\begin{tabular}{|c|c|c|c|c|c|}
\hline Sitio & Localidad & Municipio & $\begin{array}{l}\text { Co o r d e n a d a s } \\
\text { geográficas }\end{array}$ & $\begin{array}{l}\text { Altitud } \\
\text { (msnm) }\end{array}$ & Tipo de vegetación \\
\hline 1 & Barra de Cahoacán & Tapachula & $\begin{array}{l}14^{\circ} 39^{\prime} 16^{\prime \prime} \mathrm{N} \\
92^{\circ} 21^{\prime} 23 ” \mathrm{~W}\end{array}$ & 10 & Manglar \\
\hline 2 & San Antonio & Huehuetán & $\begin{array}{l}14^{\circ} 59^{\prime} 53^{\prime \prime} \mathrm{N} \\
92^{\circ} 26^{\prime} 25^{\prime \prime} \mathrm{W}\end{array}$ & 50 & Cacaotal \\
\hline 3 & Izapa Segunda Sección & Tuxtla Chico & $\begin{array}{l}14^{\circ} 53^{\prime} 00^{\prime \prime} \mathrm{N} \\
92^{\circ} 10^{\prime} 10^{\prime \prime} \mathrm{W}\end{array}$ & 260 & Cacaotal \\
\hline 4 & Rosario Izapa & Tuxtla Chico & $\begin{array}{l}14^{\circ} 58^{\prime} 30^{\prime \prime} \mathrm{N} \\
92^{\circ} 09^{\prime} 30^{\prime \prime} \mathrm{W}\end{array}$ & 500 & Cafetal de sombra \\
\hline 5 & Unión Roja & Cacahoatán & $\begin{array}{l}15^{\circ} 02^{\prime} 43^{\prime \prime} \mathrm{N} \\
92^{\circ} 13^{\prime} 01^{\prime \prime} \mathrm{W}\end{array}$ & 600 & Cafetal de sombra \\
\hline 6 & El Águila & Cacahoatán & $\begin{array}{l}15^{\circ} 05^{\prime} 31^{\prime \prime} \mathrm{N} \\
92^{\circ} 11^{\prime} 00^{\prime \prime} \mathrm{W}\end{array}$ & 1200 & Cafetal de sombra \\
\hline 7 & Benito Juárez El Plan & Cacahoatán & $\begin{array}{l}15^{\circ} 05^{\prime} 11^{\prime \prime} \mathrm{N} \\
92^{\circ} 08^{\prime} 52^{\prime \prime} \mathrm{W}\end{array}$ & 1500 & $\begin{array}{l}\text { Bosque mesófilo de } \\
\text { montaña }\end{array}$ \\
\hline 8 & Chiquihuites & Unión Juárez & $\begin{array}{l}15^{\circ} 05^{\prime} 40^{\prime \prime} \mathrm{N} \\
92^{\circ} 06^{\prime} 30^{\prime \prime} \mathrm{W}\end{array}$ & 2200 & $\begin{array}{l}\text { Bosque mesófilo de } \\
\text { montaña }\end{array}$ \\
\hline 9 & Papales & Cacahoatán & $\begin{array}{l}15^{\circ} 07^{\prime} 00^{\prime \prime} \mathrm{N} \\
92^{\circ} 07^{\prime} 30^{\prime \prime} \mathrm{W}\end{array}$ & 3000 & $\begin{array}{l}\text { Bosque de pino- } \\
\text { encino }\end{array}$ \\
\hline
\end{tabular}


Cuadro 2. Especies registradas en los sitios estudiados del gradiente altitudinal. Los números asignados a cada sitio son los mismos que los presentados en el Cuadro 1. Los microhábitats para las especies fueron: S, subcortícola; A, alboduramícola; I, interfase suelo-tronco.

\begin{tabular}{|c|c|c|c|c|c|c|c|c|c|c|c|c|c|c|c|c|}
\hline \multirow{2}{*}{ Especie } & \multicolumn{9}{|c|}{ Sitio } & \multicolumn{4}{|c|}{$\begin{array}{|ll|}\text { Estado } & \text { de } \\
\text { descomposición } & \\
\end{array}$} & \multicolumn{3}{|c|}{ Microhábitat } \\
\hline & 1 & 2 & 3 & 4 & 5 & 6 & 7 & 8 & 9 & I & II & III & IV & $\mathbf{S}$ & $\mathbf{A}$ & I \\
\hline $\begin{array}{l}\text { Passalus (Passalus) } \\
\text { punctiger Lepeletier y } \\
\text { Serville, } 1825\end{array}$ & & 2 & 1 & 2 & 2 & & & & & 1 & 3 & 3 & & & 6 & 1 \\
\hline $\begin{array}{l}\text { Passalus (Pertinax) caelatus } \\
\text { Erichson, } 1847\end{array}$ & & & & & & 6 & & & & & 2 & 4 & & 2 & 4 & \\
\hline $\begin{array}{l}\text { Passalus (Pertinax) } \\
\text { punctatostriatus Percheron, } \\
1835\end{array}$ & & & & 5 & & & 1 & & & & & 6 & & & 6 & \\
\hline $\begin{array}{l}\text { Paxillus leachi Macleay, } \\
1819\end{array}$ & & & & & 2 & & & & & & 2 & & & & 2 & \\
\hline $\begin{array}{l}\text { Chondrocephalus debilis } \\
\text { (Bates, 1886) }\end{array}$ & & & & & & & & 13 & & & 2 & 7 & 4 & & 13 & \\
\hline $\begin{array}{l}\text { Chondrocephalus gemmae } \\
\text { Reyes-Castillo y Castillo, } \\
1986\end{array}$ & & & & & & & & 1 & 6 & & 5 & 2 & & 2 & 5 & \\
\hline $\begin{array}{l}\text { Chondrocephalus } \\
\text { granulifrons (Bates, 1886) }\end{array}$ & & & & & & & & 47 & 1 & & & 1 & 47 & & 48 & \\
\hline Heliscus sp & & & & & & & 25 & & & & & 25 & & & 25 & \\
\hline $\begin{array}{l}\text { Odontotaenius } \\
\text { striatopunctatus (Percheron, } \\
1835 \text { ) }\end{array}$ & & & 18 & & 3 & & & & & 11 & 3 & 7 & & & 21 & \\
\hline Oileus sargi (Kaup, 1871) & & & & & & & 5 & & & & 3 & 2 & & & 5 & \\
\hline $\begin{array}{l}\text { Pseudacanthus junctistriatus } \\
\text { (Kuwert, 1891) }\end{array}$ & & & & & & & & 5 & 8 & & 6 & 7 & & & 13 & \\
\hline $\begin{array}{l}\text { Pseudacanthus subopacus } \\
\text { (Bates, 1886) }\end{array}$ & & & & & & & & 6 & & & & 1 & 5 & & 6 & \\
\hline $\begin{array}{l}\text { Undulifer nigidioides } \\
\text { (Hincks, 1949) }\end{array}$ & & & & & & & 2 & & & & & 2 & & & 2 & \\
\hline $\begin{array}{l}\text { Vindex synelytris Gravely, } \\
1918\end{array}$ & & & & & & & & & 2 & & & 2 & & & 2 & \\
\hline Vindex sp. & & & & & & & 10 & 5 & & & 8 & 7 & & & 15 & \\
\hline No. especies & $\mathbf{0}$ & 1 & 2 & 2 & 3 & 1 & 5 & 6 & 4 & 2 & 9 & 14 & 3 & 2 & 15 & 1 \\
\hline No. individuos & $\mathbf{0}$ & 2 & 19 & 7 & 7 & 6 & 43 & 77 & 17 & 12 & 34 & 76 & 56 & 4 & 173 & 1 \\
\hline
\end{tabular}


Cuadro 3. Disponibilidad de madera y promedio $( \pm D E)$ de las variables del tronco en los sitios del gradiente altitudinal en el Soconusco. Los números asignados a cada sitio se indican en el Cuadro 1.

\begin{tabular}{|c|c|c|c|c|c|c|}
\hline Sitio & $\begin{array}{l}\text { N ú m e r o } \\
\text { de madera } \\
\text { muerta }\end{array}$ & 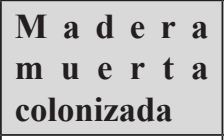 & $\begin{array}{l}\text { \% de madera } \\
\text { colonizada }\end{array}$ & 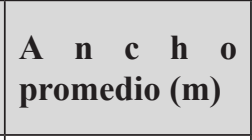 & $\begin{array}{llll}\mathbf{L} & \mathbf{a} & \mathbf{r} & \mathbf{g} \\
\text { promedio } & \mathbf{( m )}\end{array}$ & $\begin{array}{l}\text { V o l u m e n } \\
\text { promedio }\left(\mathrm{m}^{3}\right)\end{array}$ \\
\hline 1 & 39 & 0 & 0 & $0.09 \pm 0.08$ & $1.31 \pm 1.26$ & $0.01 \pm 0.02$ \\
\hline 2 & 38 & 2 & 5.2 & $0.12 \pm 0.13$ & $1.25 \pm 0.71$ & $0.02 \pm 0.05$ \\
\hline 3 & 24 & 5 & 20.8 & $0.16 \pm 0.15$ & $1.20 \pm 0.73$ & $0.05 \pm 0.14$ \\
\hline 4 & 15 & 4 & 26.6 & $0.13 \pm 0.14$ & $2.50 \pm 2.07$ & $0.11 \pm 0.24$ \\
\hline 5 & 13 & 1 & 7.6 & $0.09 \pm 0.06$ & $1.59 \pm 0.79$ & $0.01 \pm 0.02$ \\
\hline 6 & 34 & 3 & 8.8 & $0.08 \pm 0.06$ & $1.65 \pm 1.56$ & $0.01 \pm 0.03$ \\
\hline 7 & 21 & 5 & 23.8 & $0.18 \pm 0.14$ & $2.52 \pm 1.70$ & $0.10 \pm 0.16$ \\
\hline 8 & 15 & 7 & 46.6 & $0.22 \pm 0.19$ & $2.31 \pm 2.54$ & $0.17 \pm 0.41$ \\
\hline 9 & 25 & 4 & 16 & $0.09 \pm 0.05$ & $1.64 \pm 1.40$ & $0.01 \pm 0.01$ \\
\hline Promedio & $24.88 \pm 3.44$ & $3.44 \pm 2.18$ & 13.8 & $0.12 \pm 0.12$ & $1.65 \pm 1.47$ & $0.04 \pm 0.15$ \\
\hline
\end{tabular}

